\title{
Advanced Liver Fibrosis in Peruvian Patients with Type 2 Diabetes Mellitus: A cross-sectional Study
}

\author{
Friné Izaguirre $^{1}$, Valerie Sandra Mayorga ${ }^{1}$, Gillian Ayleen Mayorga ${ }^{1}$, Jorge Garavito ${ }^{1,2}$, Katty Manrique ${ }^{1,3}$ and \\ Adelina Lozano ${ }^{1,2^{*}}$
}

${ }^{1}$ Faculty of Medicine, Universidad Peruana Cayetano Heredia, Peru

${ }^{2}$ Department of Gastroenterology, Hospital Nacional Arzobispo Loayza, Peru

${ }^{3}$ Department of Endocrinology, Hospital Nacional Arzobispo Loayza, Peru

Submission: March 25, 2020; Published: April 09, 2020

*Corresponding author: Adelina Lozano, Associate Professor and Consultant Gastroenterology Hepatology Unit, Department of Gastroenterology, Hospital Nacional Arzobispo Loayza, 848 Alfonso Ugarte Ave., Lima 01, Lima, Peru

\begin{abstract}
Background: Non-alcoholic fatty liver disease is highly prevalent among patients with type 2 diabetes mellitus. It can progress to liver fibrosis and ultimately cirrhosis. The aim of this study was to assess frequency of liver fibrosis in Peruvian patients with type 2 diabetes mellitus and describe factors associated to advanced liver fibrosis.

Methods: We included Peruvian patients with type 2 diabetes mellitus from the endocrinology outpatient clinic at a tertiary care center. Demographic, clinical and laboratory data was obtained from all subjects. Transient elastography was used for the assessment of liver fibrosis using whether an M or XL probe. Liver stiffness measurement was splitted in two (no advanced fibrosis vs advanced fibrosis) and three (none or mild fibrosis/ intermediate probability of advanced fibrosis/ advanced fibrosis) categories, using cut-off values suggested by recent literature.

Results: Data from 100 participants was analysed. $67 \%$ were women and $28 \%$ were obese. Advanced liver fibrosis frequency was $13 \%$. On univariate analysis higher level of AST, ALT and GGT were correlated with advanced fibrosis. GGT was the only variable independently associated to advanced fibrosis $(\mathrm{p}<0.05)$.

Conclusions: Advanced liver fibrosis assessed by transient elastography is highly frequent among Peruvian patients with type 2 diabetes Mellitus.

Keywords: Liver fibrosis; Type 2 diabetes mellitus; Elastography; Non-alcoholic fatty liver disease; Hepatocellular carcinoma; Liver biopsies; Hepatitis C virus

Abbreviations: NAFLD: Non-Alcoholic Fatty Liver Disease; LSM: Liver Stiffness; T2DM: Type 2 Diabetes Mellitus; NASH: Non-Alcoholic Steatohepatitis; NAFL: Non-Alcoholic Fatty Liver; HDL: High Density Lipoprotein; LDL: Low Density Lipoprotein, AST: Aspartate Aminotransferase, ALT: Alanine Aminotransferases and GGT: Gamma-Glutamyl Transferase; CAP: Controlled Attenuation Parameter
\end{abstract}

\section{Introduction}

Non-alcoholic fatty liver disease (NAFLD) is a very common condition, with a global prevalence around 25.24\%[1]. This number is increased in high risk populations such as patients with type 2 diabetes mellitus (T2DM), with two thirds of them being affected by this disease [1]. NAFLD encompasses a spectrum of pathological conditions ranging from non-alcoholic fatty liver (NAFL) to non-alcoholic steatohepatitis (NASH), liver fibrosis and ultimately cirrhosis [2]. Patients with T2DM are more susceptible to severe forms of NAFLD and have a higher progression to hepatocellular carcinoma so concern has been raised about the link between T2DM and liver disease [3].
Patients with T2DM and liver fibrosis are usually asymptomatic and are sometimes overlooked on routine medical visits. There is a lack of consensus regarding liver disease screening on these patients [1,4]. Liver biochemistries, which are routinely used, can be normal in patients with NAFLD and are not sufficiently sensitive to serve as screening tests [1]. Ultrasound on the other hand, is not invasive and can show fatty liver disease but has a low sensitivity in identifying fibrosis [5]. Liver fibrosis markers such as NAFLD Fibrosis Score, AST/ALT ratio, FIB4 index and BAAD score are used to estimate the degree of liver fibrosis in NAFLD patients non-invasively and have shown excellent diagnostic performance 
in different studies. However, the clinical significance of the markers should be validated in different cohorts [6]. Biopsy is the gold standard for the diagnosis NAFLD and fibrosis, but, its high cost, sampling bias and invasive nature limit its use [2]. Transient elastography (TE) is a non-invasive technique that measures liver stiffness (LSM) in kilopascals ( $\mathrm{kPa})$, a parameter that strongly correlates to the degree of liver fibrosis, specially advanced fibrosis, as evaluated by simultaneous liver biopsies [2].

In the last years, several studies were conducted in order to estimate frequency of liver fibrosis in different populations with T2DM by means of transient elastography, with some alarming numbers as high as $21 \%$ [7]. These studies also described the clinical/demographic characteristics and laboratory data associated to advanced fibrosis. However, there is not many literature published on Latin American population, which is at high risk for liver fibrosis [8]. The prevalence of diabetes in Peru is $7 \%$, and $8.4 \%$ in its capital, Lima, a value that has almost doubled in the last seven years [9]. With this, the incidence of chronic complications of T2DM such as liver disease is also expected to increase [10]. Given that rapid growth of the overall prevalence of diabetes in Peru and the lack of literature published about liver disease on Latin American population with T2DM, we decided to assess the frequency of liver fibrosis and describe factors associated to advanced fibrosis in Peruvian patients with type 2 diabetes mellitus in a tertiary care center using transient elastography.

\section{Methods}

\section{Subjects}

This is a cross-sectional study conducted at the Endocrinology Outpatient Clinic in a tertiary referral hospital in Lima Peru. Patients 18 years old or older with known story of type 2 Diabetes Mellitus and who were willing to participate were consecutively admitted. Subjects with positive hepatitis B surface antigen, hepatitis $\mathrm{C}$ virus antibodies and/or Antinuclear antibody titers $>1 / 16010$, history of known chronic liver disease, chronic use of any steatogenic drug (e.g., amioradone, methotrexate, glucocorticoids, estrogens) and alcohol consumption ( $>21$ standard drinks per week in men and $>14$ standard drinks per week in women in the last 2-years) [1] were excluded. Patient history was taken and a physical examination was performed to document demographic (e.g., gender, age) and clinical (e.g., time of T2DM, waist circumference, body mass index) data. Blood samples were collected after overnight fasting for triglycerides, total cholesterol, (HDL), low density lipoprotein cholesterol (LDL), aspartate aminotransferases (AST), alanine aminotransferases (ALT) and gamma-glutamyl transferase (GGT). Written informed consent was obtained from all the enrolled patients. Local ethics committee (Universidad Peruana Cayetano Heredia, Lima, Peru) approved the study protocol.

\section{Liver fibrosis assessment}

Two experienced operators, with more than 500 measurements each, performed LSM using transient elastography (Fibroscan) in fasting patients. An M probe was located in the right upper quadrant through the intercostal spaces with the patients in a dorsal decubitus position and the right arm in maximal abduction. When M probe failed, an XL probe was used. LSM was expressed in $\mathrm{kPa}$ and reliable measurements were considered as the median of 10 valid shots, with interquartile range to median LSM ratio $<30 \%$ and success rate $\geq 60 \%$ [11].

A cut off value for advanced fibrosis was established as proposed by Wong et al, $\geq 9.6 \mathrm{kPa}$ and $\geq 9.3 \mathrm{kPa}$ for $\mathrm{M}$ and XL probes respectively. This cut off value allowed us to divide the degree of fibrosis in two groups: no advanced fibrosis $(<9.6 /<9.3 \mathrm{kPa})$ and advanced fibrosis $(\geq 9.6 / \geq 9.3 \mathrm{kPa})$ [12]. Additionally a cut off value of $<7.9 \mathrm{kPa}$ (M probe) and $<7.2 \mathrm{kPa}$ (XL probe) was used in order to divide the degree of fibrosis in three groups, as suggested by Castera: Advanced fibrosis $(>9.6 />9.3 \mathrm{kPa})$, intermediate probability of advanced fibrosis (7.9-9.6kPa/7.2$9.3 \mathrm{kPa})$ and none or mild fibrosis $(<7.9 \mathrm{kPa} / 7.2 \mathrm{kPa})$ [13]. Patients with measurements $\geq 7.9 / 7.2 \mathrm{kPa}$ were referred to the gastroenterologist for further evaluation and/or management [13].

\section{Statistical analysis}

We summarized the data using mean \pm standard deviation for normally distributed variables and median and interquartile range for skewed variables. Categorical data was expressed as percentage and counts. We used T-Student and Mann-Whitney $\mathrm{U}$ for numerical variables when continuous data was separated in two groups and ANOVA or Kruskall-Wallis if continuous data was split in three groups. Chi square was used for categorical data assessment. Multivariate analysis using logistic regression was performed in variables that were significant on univariate analysis. A p value $<0.05$ was considered statistically significant and $95 \%$ confidence intervals were calculated for each predictive test. All analysis were performed using SPSS software, version 24.0 (IBM SPSS Statistics).

\section{Results}

A total of 110 patients were invited to participate during two weeks in February 2019. 8 patients refused participate, one had positive antibodies for Hepatitis $\mathrm{C}$ virus and one had an unreliable measurement on TE resulting in 100 patients (90\%) that were included in the final analysis. Characteristics of these subjects are summarized in table 1 . The mean age of patients was 61.77 \pm 10.9 , most of them being women ( $67 \%$ vs $33 \%)$. Mean waist circumference was $98.14 \pm 11.76$, mean body mass index was $28.148 \pm 5.41$ and $28 \%$ were obese. Based on the proposed cut-off for liver stiffness measurement the frequency of advanced fibrosis was $13 \%$. 


\section{Advanced Research in Gastroenterology \& Hepatology}

Table 1: Main characteristics of patients.

\begin{tabular}{|c|c|}
\hline Variable & $n=100$ \\
\hline \multicolumn{2}{|l|}{ Demographics } \\
\hline \multicolumn{2}{|l|}{ Gender } \\
\hline Female n (\%) & $67(67 \%)$ \\
\hline Male n (\%) & $33(33 \%)$ \\
\hline Age (years) & $61.77 \pm 10.9$ \\
\hline Time of T2DM (years) & $11.19 \pm 9.84$ \\
\hline \multicolumn{2}{|l|}{ Anthropometrics } \\
\hline Waist circumference $(\mathrm{cm})$ & $98.14 \pm 11.76$ \\
\hline BMI (kg/m2) & $28.148 \pm 5.41$ \\
\hline \multicolumn{2}{|l|}{ Obesity } \\
\hline Non-obese n (\%) & $72(72 \%)$ \\
\hline Obese n (\%) & $28(28 \%)$ \\
\hline \multicolumn{2}{|l|}{ Laboratory findings } \\
\hline Triglycerides (mg/dL) & $163.23 \pm 104.685$ \\
\hline
\end{tabular}

\begin{tabular}{|c|c|}
\hline Total cholesterol $(\mathrm{mg} / \mathrm{dL})$ & $181.11 \pm 51.838$ \\
\hline HDL cholesterol $(\mathrm{mg} / \mathrm{dL})$ & $45.40 \pm 14.008$ \\
\hline LDL cholesterol $(\mathrm{mg} / \mathrm{dL})$ & $111.51 \pm 40.229$ \\
\hline AST $(\mathrm{IU} / \mathrm{L})$ & $25.65 \pm 16.010$ \\
\hline ALT $(\mathrm{IU} / \mathrm{L})$ & $28.81 \pm 19.043$ \\
\hline GGT (IU/L) & $43.84 \pm 55.105$ \\
\hline Advanced Fibrosis (F3-4) & $13(13 \%)$ \\
\hline
\end{tabular}

Note: BMI: Body Mass Index; HDL: High Density Lipoprotein; LDL: Low Density Lipoprotein, AST: Aspartate Aminotransferase, ALT: Alanine Aminotransferases and GGT: Gamma-Glutamyl Transferase.

When LSM was divided in two groups, patients with advanced fibrosis showed a bigger waist circumference (97.56 \pm 10.718 vs $101.92 \pm 17.3$ ), more frequency of obesity (53.8\% vs $24.1 \%$ ), lower triglycerides (150 \pm 94 vs $110 \pm 93)$ and higher values of AST ( $20 \pm 9$ vs $38 \pm 39)$, ALT ( $20 \pm 15$ vs $39 \pm 23$ ) and GGT ( $24 \pm 18$ vs $95 \pm 129$ ) (Table 2). When LSM was arranged in three groups, patients with advanced fibrosis showed lower values of total cholesterol and LDL cholesterol, and higher levels of AST, ALT and GGT (Table 3).

Table 2: Univariate analysis on factors associated with advanced fibrosis (two analysis groups).

\begin{tabular}{|c|c|c|c|}
\hline Variable & No Advanced Fibrosis $\dagger \mathbf{n = 8 7}$ & Advanced Fibrosis $\dagger \mathbf{n = 1 3}$ & $\mathbf{p}$ \\
\hline Gender (female/male) & $58(66.7 \%) / 29(33.3 \%)$ & $9(69.2 \%) / 4(30.8 \%)$ & 0.854 \\
\hline Age (years) & $61.71 \pm 10.886$ & $62.15 \pm 11.481$ & 0.668 \\
\hline Time of T2DM (years) & $8.0 \pm 14.0$ & $12.0 \pm 8.5$ & 0.361 \\
\hline Waist circumference (cm) & $97.56 \pm 10.718$ & $101.92 \pm 17.30$ & 0.008 \\
\hline BMI (kg/m) & $27.34 \pm 5.25$ & $70.46 \pm 14.64$ & 0.364 \\
\hline Obesity (yes/no) & $21(24.1 \%) / 66(75.9 \%)$ & $110.0 \pm 93.0$ & 0.026 \\
\hline Triglycerides (mg/dL) & $150.0 \pm 94.0$ & $156.0 \pm 63.0$ & 0.075 \\
\hline Total cholesterol (mg/dL) & $182.0 \pm 60.0$ & $46.0 \pm 30.0$ & 0.46 \\
\hline HDL cholesterol (mg/dL) & $42.0 \pm 15.00$ & $90.85 \pm 26.49$ & 0.052 \\
\hline LDL cholesterol (mg/dL) & $114.60 \pm 41.12$ & $38.00 \pm 39.0$ & 0.001 \\
\hline AST (IU/L) & $20.00 \pm 9.00$ & $39.00 \pm 23.0$ & 0.001 \\
\hline ALT (IU/L) & $20.00 \pm 15.0$ & $95.0 \pm 129.0$ & 0 \\
\hline GGT (IU/L) & $24.00 \pm 18.0$ & $7(53.8 \%) / 6(46.2 \%)$ & 0.366 \\
\hline AST/ALT (<1/>1) & $58(66.7 \%) / 29(33.3 \%)$ & & 0 \\
\hline
\end{tabular}

†Cut-off values were established as proposed by Wong et al., $>9.6 \mathrm{kPa}$ for $\mathrm{M}$ probe and $>9.3 \mathrm{kPa}$ for $\mathrm{XL}$ probe. Abbreviations: BMI: Body Mass Index; HDL: High Density Lipoprotein; LDL: Low Density Lipoprotein; AST: Aspartate Aminotransferase; ALT: Alanine Aminotransferases; GGT: Gamma-Glutamyl Transferase.

Table 3: Univariate analysis on factors associated with advanced fibrosis (three analysis groups).

\begin{tabular}{|c|c|c|c|c|}
\hline Variable & $\begin{array}{c}\text { None or Mild Fibrosis } \dagger \\
\mathbf{n = 8 2}\end{array}$ & $\begin{array}{c}\text { Intermediate Probability of Ad- } \\
\text { vanced Fibrosis } \dagger \mathbf{n = 5}\end{array}$ & Advance Fibrosis $\dagger \mathbf{n = 1 3}$ & $\mathbf{p}$ \\
\hline Gender (female/male) & $55(82.1 \%) / 27(81.8 \%)$ & $3(4.5 \%) / 2(6.1 \%)$ & $9(13.4 \%) / 4(12.1 \%)$ & 0.932 \\
\hline Age (years) & $61.5(13)$ & $62(15)$ & $64(11)$ & 0.69 \\
\hline Time of T2DM (years) & $7.5(14.0)$ & $14.0(23.9)$ & $12.0(8.5)$ & 0.58 \\
\hline Waist circumference (cm) & $97.7(10.63)$ & $95.7(13.34)$ & $101.9(17.31)$ & 0.44 \\
\hline BMI $\left(\mathrm{kg} / \mathrm{m}^{2}\right)$ & $27.27(4.94)$ & $28.48(10.02)$ & $30.47(14.64)$ & 0.64 \\
\hline
\end{tabular}




\section{Advanced Research in Gastroenterology \& Hepatology}

\begin{tabular}{|c|c|c|c|c|}
\hline Obesity (yes/no) & $\begin{array}{c}20(24.4 \%) / 62 \text { (75.6 } \\
\%)\end{array}$ & $1(20.0 \%) / 4(80.0 \%)$ & 7 (53.8 \%) / 6 (46.2\%) & 0.82 \\
\hline Triglycerides (mg/dL) & $152(101)$ & $150(-)$ & $110(82)$ & 0.88 \\
\hline Total cholesterol (mg/dL) & $183(64)$ & $113(-)$ & $145(65)$ & 0.01 \\
\hline HDL cholesterol (mg/dL) & $41(14)$ & $38(-)$ & $46(33)$ & 0.32 \\
\hline LDL cholesterol (mg/dL) & $117.39(43.31)$ & $74.33(7.37)$ & $90.67(32.11)$ & 0.04 \\
\hline AST (IU/L) & $20(9)$ & $29(-)$ & $38(39)$ & 0 \\
\hline ALT (IU/L) & $20(15)$ & $32(-)$ & $41(23)$ & 0 \\
\hline GGT (IU/L) & $24(15)$ & $50(-)$ & $102(211)$ & 0 \\
\hline AST/ALT $(<1 />1)$ & $54(65.9 \%) / 28(34.1 \%)$ & $4(80 \%) / 1(20 \%)$ & $7(53.8 \%) / 6(46.2 \%)$ & 0.54 \\
\hline
\end{tabular}

Note: $†$ Cut-off values were established as proposed by Castera using M and XL probe: advanced fibrosis (>9.6/>9.3kPa), intermediate probability of advanced fibrosis (7.9-9.6kPa/7.2-9.3kPa) and none or mild fibrosis (<7.9kPa/7.2kPa). Abbreviations: BMI: Body Mass Index; HDL: High

Density Lipoprotein; LDL: Low Density Lipoprotein; AST: Aspartate Aminotransferase; ALT: Alanine Aminotransferases; GGT: GammaGlutamyl Transferase

AST, ALT, GGT showed persistent association with advanced fibrosis in both analysis. These variables were tested on multivariate analysis with the LSM measurements divided in two and three groups (Table 4). In both, GGT was the only variable that was independently related to advanced fibrosis $(\mathrm{p}=0.000$, 95\%CI $=0.002-0.004 / \mathrm{p}=0.000,95 \% \mathrm{CI}=0.004-0.010$ ).

Table 4: Factors associated with advanced fibrosis (Multivariate analysis)

\begin{tabular}{|c|c|c|c|}
\hline & Variable & $\mathbf{p}$ & $\mathbf{9 5 \%}$ CI \\
\hline \multirow{2}{*}{$\begin{array}{c}\text { Two analysis } \\
\text { group (Table 2) }\end{array}$} & AST & 0.104 & -0.015 \\
\cline { 2 - 4 } & ALT & 0.564 & -0.012 \\
\cline { 2 - 4 } & GGT & 0 & $0.002-0.004$ \\
\hline \multirow{2}{*}{$\begin{array}{c}\text { Three analysis } \\
\text { group (Table 3) }\end{array}$} & AST & 0.051 & $0.000-0.031$ \\
\cline { 2 - 4 } & ALT & 0.554 & -0.026 \\
\cline { 2 - 4 } & GGT & 0 & $0.004-0.010$ \\
\hline
\end{tabular}

Note: Multivariate analysis shows GGT as the only variable independently associated with advanced fibrosis when classifying data in either two or three groups. Abbreviations: BMI: Body Mass Index; HDL: High Density Lipoprotein; LDL: low density lipoprotein; AST: Aspartate Aminotransferase; ALT: Alanine Aminotransferases; GGT: Gamma-Glutamyl Transferase

\section{Discussion}

We found a $13 \%$ frequency of advanced liver fibrosis $(\geq 9.6 / \geq 9.3 \mathrm{kPa})$ among Peruvian patients with T2DM. This is similar to the frequency found by means of NAFLD fibrosis score in patients with T2DM in Chile, which was $12.8 \%$ [14]. However, our findings are two times higher than the frequency described by Roulot in French patients with T2DM using transient elastography, in which $7.3 \%$ had advanced liver fibrosis ( $\geq 9.6 / \geq 9.3 \mathrm{kPa}$ ) [15]. On the other hand, studies in Asian populations that used transient elastography and with the same cut off value for advanced liver fibrosis as our study have shown higher frequencies: Turkey (16.9\%), Hong Kong (17.7\%) and Malaysia (21\%) [7,16,17]. Studies establish differences in prevalence and also severity of NAFLD among different ethnicities. Latin American population has shown higher prevalence and severity of NAFLD compared to Caucasian population [8]. In the same way, Asian population has been associated with severe liver steatosis and inflammation when compared with Caucasians [18]. Some other studies reported even higher frequencies of advanced liver fibrosis in patients with T2DM; however, discrepancy in advanced fibrosis definition [19] and the fact that some of them only assess fibrosis in patients with NAFLD-diagnosis $[19,20]$ unable us to make a fair comparison.

Initial approaches classified fibrosis in five stages. But, due to the association of the last two (F3, F4) with higher risk of mortality, liver fibrosis was classified in two groups: advanced and no advanced fibrosis [21]. There is no consistency in the association between clinical/demographic variables and fibrosis severity described in different literature. Age, BMI and time of T2DM has been described [15-17]. Laboratory data and its correlation with advanced fibrosis was also different among the existing researches, with Triglycerides, HDL, ALT and GGT being the more common associations with advanced liver fibrosis [6,1517]. We found a correlation between lower triglycerides levels and advanced fibrosis on the univariate analysis, similar to the results found in a Malaysian study [6]. Contrastingly, another study has shown an association with high triglyceride levels [17]. This could be due to insulin failure to appropriately suppress hepatic VLDL secretion [3]. However, over time, hepatocellular injury and fibrosis disrupt VLDL production which could lead to an inverse relationship between triglyceride and fibrosis levels [22].

Another strategy suggests to classify patients into three groups, so that LSM is divided into No/Mild fibrosis, advanced fibrosis and a third group between these two corresponding to patients whose results needs further evaluation by means of liver biopsy [13]. We used this approach in order to assess characteristics of this third group. However, none of the studies reviewed used this strategy.

In both analysis (two groups and three groups of liver fibrosis), ALT, AST and GGT showed correlation to advanced fibrosis. Despite this, the AST to ALT ratio, which is a fibrosis biomarker commonly used in clinical practice [6], did not show association with advanced liver fibrosis. Figure 1 shows ALT, AST and GGT 
distribution in three stages of fibrosis. Only GGT levels correlate with fibrosis severity. This was also the only variable associated independently with liver fibrosis on multivariate analysis. We found two similar studies that included GGT as a variable. Both showed an independent association of GGT with advanced fibrosis $[7,15]$. GGT is increased in most patients with NASH in response to low levels of intracellular glutathione, a consequence of free radical generation in fatty liver disease [23]. However, the pathogenesis of GGT as marker for advanced liver fibrosis in patients with T2DM needs to be further evaluated. Due to the association between GGT and liver fibrosis progression found in our study, TE and liver biopsy appears to be advisable in T2DM patients with high levels of GGT.

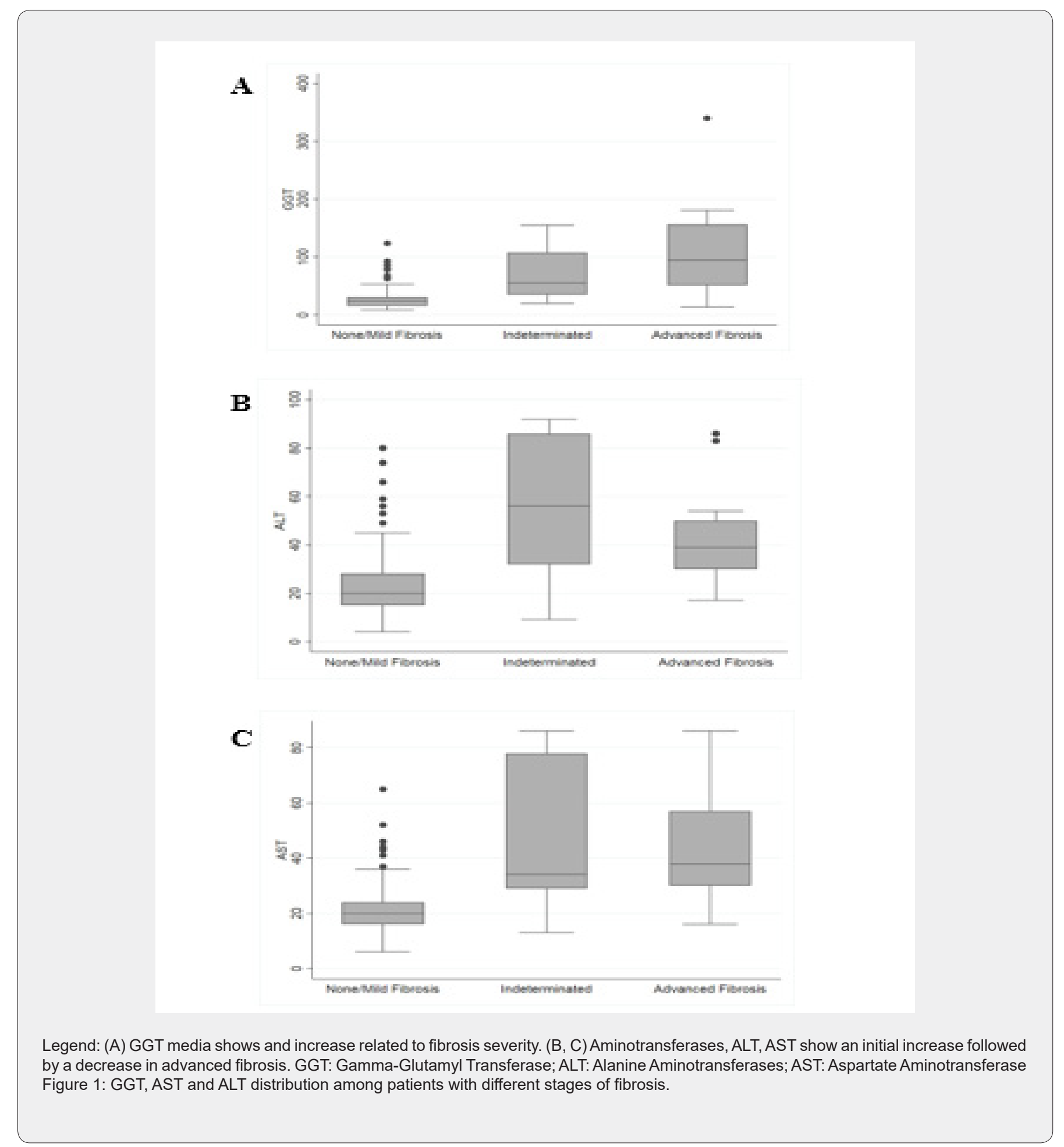


According to recent studies, prevalence of diabetes mellitus in Peru is about $7 \%$, and $8.4 \%$ in its capital, Lima, a value that has almost doubled in the last seven years [9]. It is almost sure that this will continue to increase in the future. With this raise, chronic complications of T2DM such as liver disease will become more prevalent. Current national guidelines suggest the use of aminotransferases to screen for liver disease patients with type 2 diabetes [24]. However, a decrease in aminotransferases levels as seen in Figure 1 could be interpreted as a liver disease improvement when in fact could be due to advanced liver fibrosis. Given the high frequency (13\%) of advanced liver fibrosis among patients with T2DM found in our study, more sensible methods of screening for liver disease should be used. Transient elastography is an excellent method for detecting advanced liver fibrosis but due to its high cost, is not readily available in all institutions. This is why noninvasive markers of fibrosis should be validated in Peruvian population [6]. As well, collaborative work should be done between endocrinology and gastroenterology departments when treating patients with diabetes mellitus, as the liver is a target organ in this disease.

Our study has some limitations. Biopsy was not performed in order to confirm advanced liver fibrosis. Controlled attenuation parameter (CAP) measurements were not obtained which did not allow us to describe the frequency of liver steatosis. In addition, laboratory tests, known for their association with advanced liver fibrosis, such as platelet count and serum albumin were not performed in this study. Serum albumin along with platelet count would have allowed us to calculate the NAFLD Fibrosis Score [6]. Also, this study was conducted in only one tertiary referral hospital in Lima, Peru, which means that our results only are applicable to the population studied. A multicentric study is needed in order to assess the prevalence of liver fibrosis in Peru and confirm the generalizability of our results. On the other hand, a cohort study on patients with T2DM is needed to establish the risk factors for advanced liver fibrosis.

To conclude, we found a high frequency of advanced liver fibrosis $(13 \%)$ by means of TE in Peruvian patients with T2DM at the Endocrinology Outpatient Clinic in a tertiary referral hospital. However, further research is needed to assess the prevalence of liver fibrosis in Peruvian patients with T2DM as well as the frequency of liver fibrosis in patients with T2DM in other parts of Latin America.

\section{Acknowledgments}

The authors thank Dr. Jorge Tarrillo, Dr. Carolina Sarria, Dr. Claudia Michahelles and Dr. Freddy Garcia for their support in the research project

\section{References}

1. Chalasani N, Younossi Z, Lavine J, Charlton M, Cusi K, et al. (2017) The diagnosis and management of nonalcoholic fatty liver disease: Practice guidance from the American Association for the Study of Liver
Diseases. Hepatology 67(1): 328-357.

2. Wong VW, Vergniol J, Wong GL, Foucher J, Chan HL, et al. (2010) Diagnosis of fibrosis and cirrhosis using liver stiffness measurement in nonalcoholic fatty liver disease. Hepatology 51(2): 454-462.

3. Bril F, Cusi K (2017) Management of Nonalcoholic Fatty Liver Disease in Patients with Type 2 Diabetes: A Call to Action. Diabetes Care 40(3): 419-430.

4. American Diabetes Association (2019) Comprehensive Medical Evaluation and Assessment of Comorbidities: Standards of Medical Care in Diabetes-2019: Diabetes Care. 42 (Suppl 1): S34-S45.

5. Khov N (2014) Bedside ultrasound in the diagnosis of nonalcoholic fatty liver disease. World J Gastroenterol 20(22): 6821-6825.

6. Enomoto H (2015) Liver fibrosis markers of nonalcoholic steatohepatitis. World J Gastroenterol 21(24): 7427-7435.

7. Lai L, Yusoff WNIW, Vethakkan SR, Mustapha NRN, Mahadeva S, et al. (2019) Screening for non-alcoholic fatty liver disease in patients with type 2 diabetes mellitus using transient elastography. J Gastroenterol Hepatol 34(8):1396-1403.

8. Saab S, Manne V, Nieto J, Schwimmer JB, Chalasani NP (2016) Nonalcoholic Fatty Liver Disease in Latinos. Clin Gastroenterol Hepatol 14(1): 5-12.

9. Seclen SN, Rosas ME, Arias AJ, Huayta E, Medina CA (2015) Prevalence of diabetes and impaired fasting glucose in Peru: report from PERUDIAB, a national urban population-based longitudinal study. BMJ Open Diabetes Res Care 3(1): 1-7.

10. Vuppalanchi R, Chalasani N (2008) Nonalcoholic fatty liver disease and nonalcoholic steatohepatitis: Selected practical issues in their evaluation and management. Hepatology 49(1): 306-317.

11. Castéra L, Foucher J, Bernard PH, Carvalho F, Allaix D, et al. (2010) Pitfalls of liver stiffness measurement: A 5-year prospective study of 13,369 examinations. Hepatology 51(3): 828-835.

12. Wong VW, Vergniol J, Wong GL, Foucher J, Chan AW, et al. (2012) Liver Stiffness Measurement Using XL Probe in Patients with Nonalcoholic Fatty Liver Disease. Am J Gastroenterol 107(12): 1862-1871.

13. Castera L (2015) Noninvasive Evaluation of Nonalcoholic Fatty Liver Disease. Semin Liver Dis 35(03): 291-303.

14. Arab JP, Barrera F, Gallego C, Valderas JP, Uribe S, et al. (2016) High prevalence of undiagnosed liver cirrhosis and advanced fibrosis in type 2 diabetic patients. Anna Hepatol 15(5): 721-728.

15. Roulot D, Roudot-Thoraval F, N Kontchou G, Kouacou N, Costes JL, et al. (2017) Concomitant screening for liver fibrosis and steatosis in French type 2 diabetic patients using Fibroscan. Liver Int 37(12): 1897-1906.

16. Demir M, Deyneli O, Yilmaz Y (2018) Screening for hepatic fibrosis and steatosis in Turkish patients with type 2 diabetes mellitus: A transient elastography study. Turk J Gastroenterol 30(3): 266-270.

17. Kwok R, Choi KC, Wong GL, Zhang Y, Chan HL, et al. (2016) Screening diabetic patients for non-alcoholic fatty liver disease with controlled attenuation parameter and liver stiffness measurements: a prospective cohort study. Gut 65(8): 1359-1368.

18. Tabibian JH, Lazo M, Durazo FA, Yeh HC, Tong MJ, et al. (2011) Nonalcoholic fatty liver disease across ethno-racial groups: Do AsianAmerican adults represent a new at-risk population? NAFLD across ethno-racial groups. J Gastroenterol Hepatol 26(3): 501-509.

19. Sporea I, Mare R, Lupusoru R, Sima A, Sirli R, et al. (2016) Liver stiffness evaluation by transient elastography in type 2 diabetes mellitus patients with ultrasound-proven steatosis. J Gastrointestin Liver Dis 25(2): 167-174. 
20. Prasetya IB, Hasan I, Wisnu W, Rumende CM (2017) Prevalence and Profile of Fibrosis in Diabetic Patients with Non-alcoholic Fatty Liver Disease and the Associated Factors. Acta Med Indones 49(2): 91-98.

21. Ekstedt M, Hagström H, Nasr P, Fredrikson M, Stål P, et al. (2015) Fibrosis stage is the strongest predictor for disease-specific mortality in NAFLD after up to 33 years of follow-up. Hepatology 61(5): 15471554 .

22. Jiang Z, Tsugawa Y, Tapper E, Lai M, Afdhal N, et al. (2015) Lowfasting triglyceride levels are associated with non-invasive markers of advanced liver fibrosis among adults in the United States. Aliment Pharmacol Ther 42(1): 106-116.
23. Whitfield JB (2001) Gamma Glutamyl Transferase. Crit Rev Clin Lab Sci 38(4): 281-290.

24. (2014) Ministerio de Salud. Guía de Práctica Clínica para la Prevención, Diagnóstico, Tratamiento y Control de la. Diabetes Mellitus Tipo 2. Lima: Ministerio de Salud, p. 18.

DOI:10.19080/ARGH.2020.15.555903 